\title{
INTEND, DEPICTION IN VITRO AND IN VIVO APPRAISAL OF GLIPIZIDE FLOATING MICROSPHERES USING ETHYL CELLLULOSE AND HYDROXYL PROPYL METHYL CELLULOSE AS POLYMER BY SUBSTANTIALLY MODIFIED METHOD
}

\author{
RISHIKESH GUPTA ${ }^{1 *}$, SK PRAJAPATI ${ }^{1}$, SNIGDHA PATTNAIK ${ }^{2}$, PEEYUSH BHARDWAJ $^{1}$ \\ ${ }^{1}$ Institute of Pharmacy, Bundelkhand University, Jhansi, Uttar Pradesh, India. ${ }^{2}$ School of Pharmaceutical Sciences, \\ Siksha 'O' Anusandhan University, Bhubaneswar, Odisha, India. Email: rishikeshgupt@gmail.com
}

Received: 05 May 2016, Revised and Accepted: 14 May 2016

\section{ABSTRACT}

Objective: The purpose of this research was to formulate and evaluate floating microsphere of glipizide.

Methods: Glipizide microsphere containing ethyl cellulose (EC) and hydroxyl propyl methyl cellulose (HPMC) were prepared by solvent evaporation method. Polymer to drug ratio affected characteristics of microspheres. Microspheres were discrete, spherical, and perforated form. The microspheres exhibited good floating property and achieved good gastric retention.

Result: In vitro performance was evaluated by the usual pharmacopoeial and other tests such as drug polymer compatibility (Fourier transform infrared scan), yield (\%), micrometric properties such as tapped density (\%). Compressibility particle size analysis (by optical microscopy), drug entrapment efficiency, surface topography (scanning electron microscope), and in vitro release study. On the basis of results, increasing the polymer ratio increased the particle size (195.6 \pm 20.24 to $200.89 \pm 16.61$ ), increased tapped density (maximum $0.29 .60 \pm 0.00037 \mathrm{HGF} 4$, batch), and decreased $\%$ compressibility $(2.13 \pm 0.188)$. Drug loaded floating microspheres were found to be float more than $12 \mathrm{hrs}$ on simulated gastric fluid (pH-1.2). Maximum drug entrapment was found in batch HGF3 (Drug:HPMC:EC) (1:1:3). Electron microscopy showed its perforated surface with hollowness. After $10 \mathrm{hrs}$, maximum release was found to be $78.0 \%$ (batch-GF1).

Conclusion: The release study was performed in simulated gastric fluid with $0.02 \%$ tween 80 . The best release result was obtained at the ratio of drug: polymer (1:1).

Keywords: Floating microspheres, Glipizide, Gastrorentensive system, In vitro release.

(C) 2016 The Authors. Published by Innovare Academic Sciences Pvt Ltd. This is an open access article under the CC BY license (http://creativecommons. org/licenses/by/4. 0/) DOI: http://dx.doi.org/10.22159/ajpcr.2016.v9i5.12621

\section{INTRODUCTION [1-10]}

The main aim of this study was to develop a newer floating drug delivery system (FDDS) of glipizide to prolong gastric retention.

The release duration of glipizide would be prolonged and the dosing frequency would shorter and improve the enhanced the bioavailability of drug. Thus, patient compliance will be better. Ray et al. prepared floating microspheres of metformin hydrochloride by non-aqueous solvent evaporation methodusing ethyl cellulose as polymer. The microspheres remain buoyant more than $8 \mathrm{hrs}$. The present study was involved the preparation and evaluation of floating microspheres of glipizide as a model drug to prolong gastric residence time. In this study, the floating microspheres were prepared by solvent evaporation method using different ratio of ethyl cellulose and hydroxypropyl methylcellulose.

Glipizide was used as a model drug. It is an oral hypoglycemic drug. It is white crystalline powder. Practically, it is insoluble in water and alcohol, sparingly soluble in acetone.

It is soluble in dichloromethane. Its molecular weight is $444.548 \mathrm{~g} / \mathrm{mol}$. Plasma half-life of glipizide is $2-5 \mathrm{hrs}$. Melting point is $208-209^{\circ} \mathrm{C}$. Its gastroretentive absorption is uniform, rapid, and essential complete. Glipizide is sulfonylureas. Sulfonylurea likely bind to adenosine triphosphate sensitive potassium-channel receptors on the pancreatic cell surface, reducing potassium conductance, and causing depolarization of the membrane. Depolarization stimulates calcium ion influx through voltage-sensitive calcium channels, raising intracellular concentrations of calcium ions, which induce the secretion of insulin. Glipizide is rapidly and completely absorbed after oral administration with a bioavailability of approximately $95 \%$. After oral administration, the drug is $98.4 \%$ bound to plasma protein.

\section{MATERIALS AND METHODS}

\section{Materials}

Glipizide was a gift sample from Micro Labs Pvt. Ltd., Kolkata. Dichloromethane, acetone, ethyl cellulose, hydroxypropyl methylcellulose and tween80 were obtained from Central Drug House, Mumbai, Maharashtra, India. All other chemicals were of analytical grade and were used as procured.

\section{Methods [11-14]}

Microspheres containing antidiabetic drug were prepared by solvent evaporation method. Briefly drug, ethyl cellulose, and hydroxypropylmethylcellulose were mixed in dichloromethane, ethanol, and acetone at 1:1:1 ratio. The slurry was slowly introduced in to $0.2 \%$ solution of sodium lauryl sulfate at $1200 \mathrm{rpm}$ by magnetic stirrer initially at room temperature. The solution was stirred for $1 \mathrm{hr}$ to allow the solvent to evaporate 12 and the microspheres were collected by filtration using whattman filter paper. The microspheres were washed with distilled water 3-4 times; the collected microspheres were dried for $1 \mathrm{hr}$ at room temperature and subsequently stored in desiccators over fused calcium chloride.

New method or substantially modified method [15]

Slow diffusion method

In this method, for the preparation of floating microspheres, dichloromethane was the major solvent used. However, its use during formulation is likely to create a hazardous environment to the biological system in order to circumvent this problem. Solvent diffusion and solvent 
evaporation method is used by employing the less toxic solvent such as ethyl acetate, acetone, and methanol to prepare the floating microspheres.

The ethyl acetate diffuse out until it reached an equilibrium concentration in the microspheres. Such a quick leaching out of the organic solvent in to the aqueous phase is responsible for including the interfacial polymer deposition at the $0 / \mathrm{W}$ interface, leading to the formation of the floating microspheres.

\section{Micrometric properties [16-18]}

Tapped density

The prepared microspheres were characterized for their micrometric properties such as the particle size, true density, tapped density, and \% compressibility index. The tapping method was adopted to calculate the tapped densities and \% compressibility index using following formula (Table 1 and Fig. 1):

$$
\text { Tapped density }=\frac{\text { Mass of microspheres }}{\text { Volume of microspheres after tapping }}
$$

$\%$ Compressibility index $=\left(1-\mathrm{V} / \mathrm{V}_{0}\right) \times 100$

Here, $\mathrm{V}$ and $\mathrm{V}_{0}$ are the volumes of the samples after and before the standard tapping, respectively.

\section{Particle size analysis}

The size of microspheres was estimated by an optical microscope (Magnus MLX-DX, Olympus, India) provided with a stage micrometer and an ocular micrometer. The mean particle size was estimated for about 200-300 particles shown in Table 1 and Fig. 1.

\section{Yield of microspheres}

The prepared microspheres were collected and weighed. The actual weight of obtained microspheres was divided by the total amount of all non-volatile material that was used for the preparation of the microspheres (Table 1 and Fig. 1).

$\%$ Yield $=\frac{\text { Actual weight of the product }}{\text { Total weight of the excipients and drug }} \times 100$

\section{Drug entrapment efficiency}

Microspheres ( $50 \mathrm{mg}$ ) were crushed in a glass mortar, and the powder microspheres were suspended in a $10 \mathrm{ml}$ of methanol. After $24 \mathrm{hrs}$, the solution was filtered, and the filtrate was analyzed The estimation of drug was carried out at the $\lambda_{\max }$ of $227 \mathrm{~nm}$ by UV double-beam spectrophotometer (Shimadzu UV-1800 series) after suitable dilution for the drug content using the following formula (Table 1 and Fig. 1):

Drug entrapment efficiency $=\frac{\text { Calculated drug content }}{\text { Theoretical drug content }} \times 100$

Scanning electron microscopy (SEM)

SEM (LEO-430, UK) was used for the morphological characterization of floating microspheres. Microspheres were mounted directly on sample stub using double-sided sticking tape and coated with gold palladium film (thickness $200 \mathrm{~nm}$ ) under reduced pressure (0.001 mmHg) (Fig. 2).

\section{Buoyancy}

Buoyancy percentage - Microspheres $50 \mathrm{mg}$ were spread over the surface of a USPXXIX dissolution apparatus (type П) filled with $900 \mathrm{ml}$ of simulated gastric fluid containing room temperature. The solution was stirred for $1 \mathrm{hr}$ to allow the solvent to evaporate [12], and the microspheres were collected by filtration using whattman filter paper. The microspheres were washed with distilled water 3-4 times, the collected microspheres were dried for $1 \mathrm{hr}$ at room temperature and subsequently stored in desiccators over fused calcium chloride $0.02 \%$ tween80 [13]. This medium was agitated with paddle rotation speed $100 \mathrm{rpm}$ for $12 \mathrm{hrs}$. After $12 \mathrm{hrs}$, the floated and settle microspheres were collected in Table 1 and Fig. 1.

\section{$\%$ Buoyancy $=\frac{\text { Weight of floating microspheres }}{\text { Initial weight of microspheres }} \times 100$}

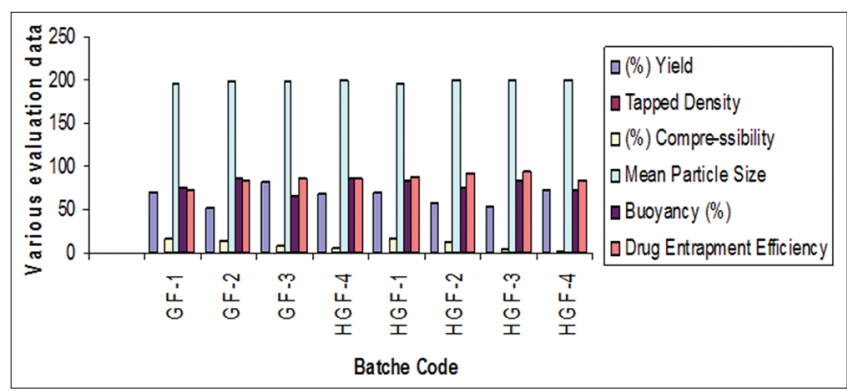

Fig. 1: Comparative study of various data

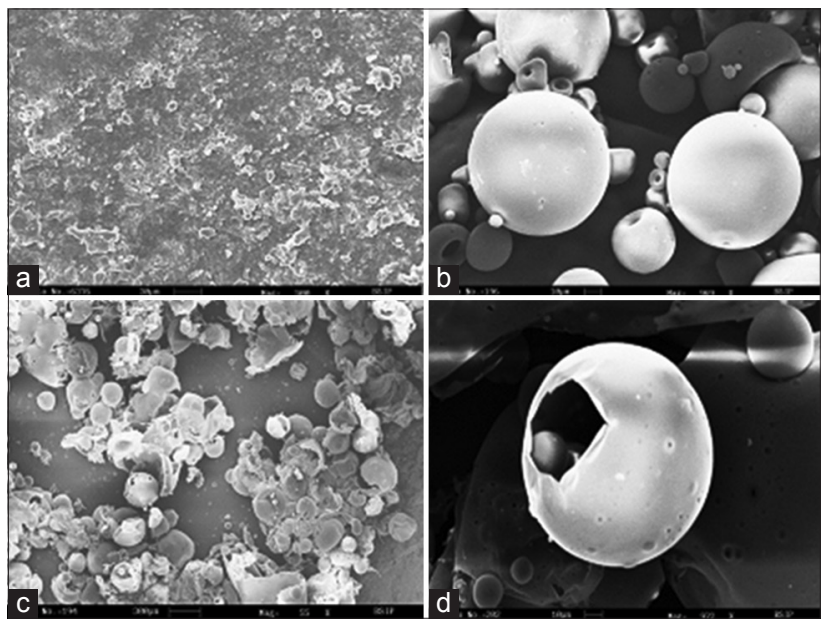

Fig. 2: Scanning electron microscopy images: (a) Surface of floating microspheres. (b) Population of floating microspheres. (c) Ruptured and roughness of microspheres. (d) Hollowness of floating microspheres

Table 1: Various formulation parameters of glipizide microsphere

\begin{tabular}{llllllc}
\hline $\begin{array}{l}\text { Batch } \\
\text { code }\end{array}$ & Yield* (\%) & $\begin{array}{l}\text { Tapped } \\
\text { density* }\end{array}$ & $\begin{array}{l}\text { Compressibility* } \\
\text { (\%) }\end{array}$ & $\begin{array}{l}\text { Mean } \\
\text { particle size* }\end{array}$ & $\begin{array}{l}\text { Buoyancy (\%)* } \\
\text { Drug entrapment } \\
\text { efficiency* }\end{array}$ & $\begin{array}{l}\text { In vitro (\%) release } \\
\text { after 10 hrs* }\end{array}$ \\
\hline GF1 & $69.1 \pm 0.83$ & $0.23 \pm 0.00094$ & $15.8 \pm 0.282$ & $195.6 \pm 20.24$ & $76.2 \pm 0.35$ & $72.5 \pm 3.3$ \\
GF2 & $51.4 \pm 1.07$ & $0.25 \pm 0.0081$ & $13.4 \pm 0.754$ & $198.5 \pm 16.1$ & $86.2 \pm 1.80$ & $84.2 \pm 1.9$ \\
GF3 & $83.0 \pm 0.41$ & $0.26 \pm 0.0012$ & $7.05 \pm 0.408$ & $197.9 \pm 17.4$ & $65.5 \pm 1.38$ & $86.9 \pm 7.8$ \\
HGF4 & $67.2 \pm 0.21$ & $0.28 \pm 0.0023$ & $5.8 \pm 0.0188$ & $199.3 \pm 16.04$ & $86.4 \pm 1.50$ & $85.3 \pm 5.2$ \\
HGF1 & $70.5 \pm 0.44$ & $0.25 \pm 0.0065$ & $15.6 \pm 0.471$ & $195.4 \pm 17.60$ & $84.9 \pm 1.17$ & $87.6 \pm 2.9$ \\
HGF2 & $57.7 \pm 0.20$ & $0.26 \pm 0.0078$ & $12.6 \pm 1.88$ & $199.6 \pm 16.00$ & $76.5 \pm 2.30$ & $91.2 \pm 1.3$ \\
HGF3 & $53.5 \pm 2.50$ & $0.28 \pm 0.0016$ & $3.66 \pm 0.471$ & $200.1 \pm 16.69$ & $83.1 \pm 1.03$ & $93.2 \pm 3.4$ \\
HGF4 & $71.4 \pm 0.48$ & $0.29 \pm 0.0037$ & $2.13 \pm 0.188$ & $200.89 \pm 1661$ & $71.8 \pm 1.40$ & $83.4 \pm 8.8$ \\
\hline
\end{tabular}

$*$ Mean \pm SD, $n=3$ (All values are the average of three determination). SD: Standard deviation 


\section{Partition coefficient}

The partition coefficient is defined as the ratio of unionized drug distributed between the organic and aqueous phase at equilibrium. For a drug delivery system, lipophilic/hydrophilic balance has been shown to be contributing factor for rate and extent of drug absorption. Partition coefficient provides a means of characterizing. Lipophilic/hydrophilic nature of drug. The measurement of drug lipophilicity and indication of its ability to cross the lipoidal cell membrane is the oil/water partition coefficient in system such as octanol/water and octanol/buffer.

The partition coefficient was calculated by the following equation:

$\mathrm{P}_{0} / \mathrm{w}=\left(\mathrm{c}_{\text {organic }} / \mathrm{c}_{\text {aqueous }}\right)$

\section{In vitro drug release}

In vitro drug release studies were performed in $0.1 \mathrm{~N} \mathrm{HCl} \mathrm{(pH} \mathrm{1.2)} \mathrm{for}$ floating microspheres. The drug release was determined in $900 \mathrm{ml}$ $0.1 \mathrm{~N} \mathrm{HCl}(\mathrm{pH} \mathrm{1.2)}$ at $100 \mathrm{rpm}$ using USP XXIII dissolution apparatus (paddle type). A weighed amount of floating microspheres equivalent to $50 \mathrm{mg}$ famotidine was placed in a non-reacting cloth having a smaller mesh size than the microspheres. The mesh was tied with a nylon thread to avoid the escape of any microspheres, and a glass bead was placed in the mesh to induce sinking of microspheres in the dissolution medium (28). The temperature of the dissolution medium was kept at $37^{\circ} \mathrm{C} \pm 0.5^{\circ} \mathrm{C}$.

At specified time intervals, $5 \mathrm{ml}$ aliquot was withdrawn, filtered, diluted with the same medium, and assayed at $227 \mathrm{~nm}$ for Famotidine using a UV double-beam spectrophotometer (Shimadzu UV-1800 series) (Table 1 and Fig. 3).

\section{Kinetics of drug release [19-21]}

The zero-order rate (Equation 1) is applicable for the systems where drug release is independent of its concentration, and it is usually seen in the dosage forms like transdermal system, osmotic system, coated tablets as well as matrix tablets with low soluble drugs. The first-order rate (Equation 2) is applicable in the systems where the release is dependent on its concentration (generally seen in case water-soluble drugs releasing from the porous matrix). The Higuchi model describes the release of the drug from an insoluble matrix to be linearly related to the square root of time and is based on Fickian diffusion (Equation 3). The Hixson-Crowell cube root law (Equation 4) explains the release of drug from the systems where it depends on the change in surface area and diameter of the particles or tablets with time and mainly applies in the case of systems that dissolute or erode over time. In order to authenticate the release model, dissolution data can further be analyzed by Peppas and Korsmeyer equation (Equation 5).

$Q_{\mathrm{t}}=\mathrm{k}_{\mathrm{n}} \mathrm{t}$

$\ln Q_{\mathrm{t}}=\ln \mathrm{Q}_{0}-\mathrm{k}_{1} \mathrm{t}$

$\mathrm{Q}_{\mathrm{t}}=\mathrm{k}_{\mathrm{HC}} \mathrm{t}^{1 / 2}$

$\mathrm{Q}_{0}^{1 / 3}-\mathrm{Q}^{1 / 3}=\mathrm{k}_{\mathrm{HC}} \mathrm{t}$

$\mathrm{M}_{\mathrm{t}} / \mathrm{M}_{\infty}=\mathrm{k} \mathrm{t}^{\mathrm{n}}$

Where, $Q_{t}$ is the amount of drug released at time t; $Q_{0}$ is the initial amount of the drug in the formulation; $\mathrm{k}_{0}, \mathrm{k}_{1}, \mathrm{k}_{\mathrm{H}}$, and $\mathrm{k}_{\mathrm{HC}}$ are release rate constants for zero-order, first-order, Higuchi model, and HixsonCrowell rate equations.

In Equation 5, $M_{t}$ is the amount of drug released at time $t$, and $M_{\infty}$ is the amount released at time infinity; $\mathrm{k}$ is the kinetic constant, and $\mathrm{n}$ is the diffusion coefficient (28).

The release data of various formulations was fitted in various models to ascertain the mechanism of drug release.

\section{In vivo study drug release [22]}

In vivo efficiency of the optimized batch GF1 was performed in healthy normal Wistar rats (250-300 g) by measuring the hypoglycemic effect produced after oral administration.

In Group A (glipizide suspension), the rapid reduction in blood glucose was observed and maximum reduction about $50 \%$ was observed within $3 \mathrm{hrs}$ after oral administration. Significant hypoglycemic effect was maintained only from 0.5 to $5 \mathrm{hrs}$.

In Group B (glipizide floating microspheres suspension), the reduction in blood glucose was sow and reaches maximum reduction about $51 \%$ was observed within 5 hrs after oral administration. Significant hypoglycemic effect (25\%) was maintained for a period of 2-12 hrs. This statistically significant hypoglycemic effect matches with Kahn and Shechter results.

Kahn and Shechter have suggested that a $25 \%$ reduction in blood glucose levels is considered a significant hypoglycemic effect.

The sustained hypoglycemic effect was observed for a longer period of time in the case of glipizide floating microspheres due to the slow release and extended absorption. This sustained release formulation was more effective than the immediate release formulation of glipizide suspension by reducing fasting plasma glucose level and side effects. The result is shown in Table 2 and Fig. 4.

\section{RESULT AND DISCUSSION}

Floating microspheres were prepared by solvent evaporation methods using hydroxyl propyl methyl cellulose (HPMC) and ethyl cellulose (EC) as polymer and constant solvent ratio. Various formulations of microspheres were prepared using gradually increase EC concentration. Total eight batches of microspheres were prepared in two groups, which were shown in Table 3. In First group, four batches were prepared with drug:EC ratio and in second group other four batches were prepared with drug:HPMC:EC ratio.

From the results, it was clear that plain and combined absorption band was not affected by drug formulation, which emphasized the

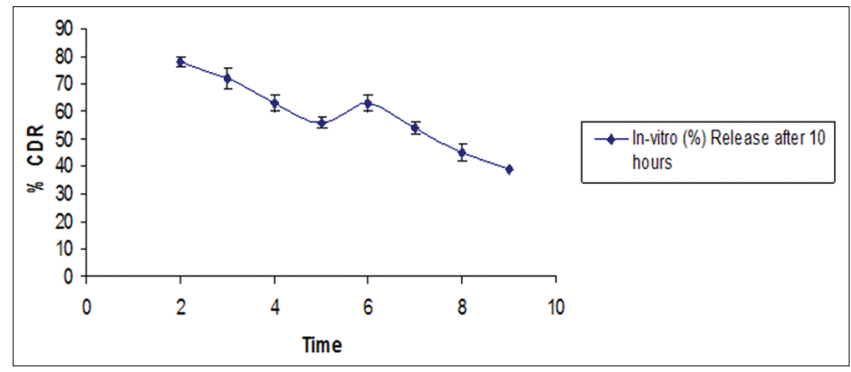

Fig. 3: In vitro (\%) drug release after $10 \mathrm{hrs}$

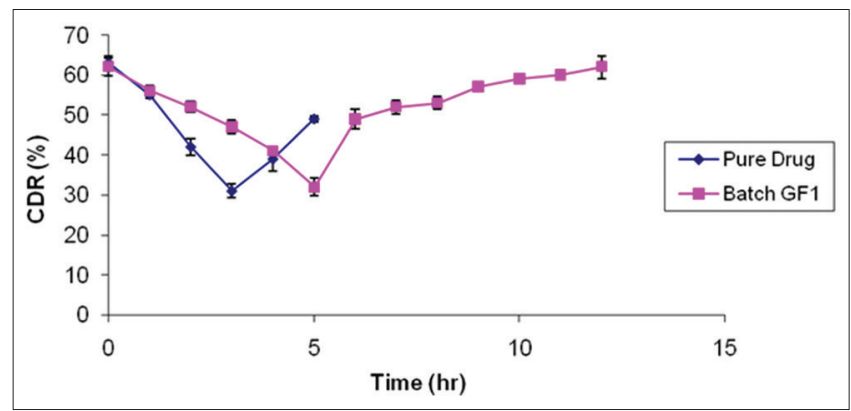

Fig. 4: In vivo comparative study of glipizide and floating microspheres (batch GF1) 
Table 2: Reduction in blood glucose level following oral administration of glipizide suspension and floating microspheres in wistar rats

\begin{tabular}{lll}
\hline Time (hrs) & $\begin{array}{l}\text { Pure } \\
\text { drug* }\end{array}$ & $\begin{array}{l}\text { Floating microspheres } \\
\text { (batch GF1)* }\end{array}$ \\
\hline 0 & $63 \pm 1.69$ & $62 \pm 2.16$ \\
1 & $55 \pm 0.83$ & $56 \pm 1.42$ \\
2 & $42 \pm 2.16$ & $52 \pm 1.41$ \\
3 & $31 \pm 1.63$ & $47 \pm 1.63$ \\
4 & $39 \pm 2.94$ & $41 \pm 0.81$ \\
5 & $49 \pm 0.80$ & $32 \pm 2.16$ \\
6 & - & $49 \pm 2.46$ \\
7 & - & $52 \pm 1.68$ \\
8 & - & $53 \pm 1.63$ \\
9 & - & $57 \pm 0.81$ \\
10 & - & $59 \pm 0.68$ \\
11 & - & $60 \pm 0.80$ \\
12 & - & $62 \pm 2.82$ \\
\hline
\end{tabular}

*Mean $(\mathrm{mg} / \mathrm{ml}) \pm \mathrm{SD}, \mathrm{n}=3$. SD: Standard deviation

Table 3: Batch specification of the prepared microspheres

\begin{tabular}{llllllll}
\hline \multirow{2}{*}{$\begin{array}{l}\text { Batch } \\
\text { code }\end{array}$} & \multicolumn{2}{l}{ Drug:polymer:polymer } & & \multicolumn{3}{c}{ Solvent ratio } \\
\cline { 2 - 3 } & Glipizide & HPMC & EC & & Ethanol & DCM & Acetone \\
\hline GF1 & 1 & 0 & 1 & & 1 & 1 & 1 \\
GF2 & 1 & 0 & 2 & & 1 & 1 & 1 \\
GF3 & 1 & 0 & 3 & & 1 & 1 & 1 \\
HGF4 & 1 & 0 & 4 & & 1 & 1 & 1 \\
HGF1 & 1 & 1 & 1 & & 1 & 1 & 1 \\
HGF2 & 1 & 1 & 2 & & 1 & 1 \\
HGF3 & 1 & 1 & 3 & 1 & 1 & 1 \\
HGF4 & 1 & 1 & 4 & 1 & 1 & 1 \\
\hline
\end{tabular}

HPMC: Hydroxyl propyl methyl cellulose, EC: Ethyl cellulose, DCM: Dichloromethane

absence of any possible interaction between the drug and polymer The plain band was similar to reference standard that confirms drug was pure.

Floating microspheres were prepared by the solvent evaporation method using HPMC and EC as polymer and constant solvent ratio which were shown in Table 3.

Microspheres were prepared using gradually increasing EC concentration. All eight batches were prepared.

First four having drug: EC ratio and last four batch having drug: HPMC: EC ratio were used. The maximum percentage yield was found to be $83.40 \pm 0.4$ (batch GF3) shown in Table 1 .

The mean particle size of the microspheres significantly increased with increasing polymer concentration and was in the range of $195.6 \pm 20.2$ to $200.08 \pm 16.6 \mu \mathrm{m}$ shown in Table 1 .

Scanning $\lambda_{\max }$ of glipizide was obtained 227 which was almost similar to standard $\lambda_{\text {max }}$ of $227 \mathrm{~nm}$. It is concluded that drug is glipizide. Drug sample gives almost straight line for graph between concentration versus absorption in methanol and buffer, which follows Beer's law; it indicates that drug sample was pure.

Partition coefficient of drug was found less than two for octanol/ simulated gastric fluid ( $\mathrm{pH}-1.2)$ and system; it concluded that drug was lipophilic in nature.

The microspheres were found to be floating for prolonged time (more than 12 hrs.) over the surface of simulated gastric fluid with $0.02 \%$ tween80 (pH-1.2).
Buoyancy percentage of microspheres was in the range $65.6 \pm 1.3 \%$ (batches GF3) to $86.4 \pm 1.5 \%$ (batch GF4), which was shown in Table 1 . The SEM photograph showed that the floating microspheres were spherical, hollow with a perforated smooth rough surface and exhibit a range of site within each batches (Fig. 2). The drug entrapment efficiency was found in the range of $72.5 \pm 3.3 \%$ (GF1) to $93.2 \pm 3.4 \%$ (batch HGF3).

The yield of microballoons was good. Microspheres showed good floating for more than 10 hours. SEM confirmed their spherical size, perforated smooth surface, and a hollow cavity in them hours.

\section{Solubility results concluded that drug was pure}

In vitro glipizide release studies were performed in simulated gastric fluid ( $\mathrm{pH}-1.2$ ) with $0.02 \%$ tween 80 for $10 \mathrm{hrs}$ (Fig. 3). The accumulative release of glipizide significantly decreased with increasing polymer concentration. In vitro drug studies were performed in Simulated Gastric Fluid (pH-1.2). Different drug release kinetics models were applied for selected batches. It was concluded that for kinetic drug release Hugichi was the best fit model.

In vivo efficiency of the optimized batch GF1 was performed in healthy normal Wistar rats (250-300 g) by measuring the hypoglycemic effect produced after oral administration. It was concluded that the sustained hypoglycemic effect was observed for a longer period of time in the case of floating microspheres due to the slow release and extended absorption, which was shown in Fig. 4

\section{CONCLUSION}

Floating microspheres offer various potential advantages for drugs with poor bioavailability because their absorption is restricted to upper gastrointestinal tract (GIT), and they can be delivered efficiently, therapy maximizing their absorption and enhancing their absolute bioavailability. Floating microspheres are retained for longer period of time than single unit dosage form.

The floating microspheres can be prepared by solvent diffusion and evaporation methods wherein the rapid diffusion solvent helps to form the hollow microspheres. Such microspheres have densities less than the density of gastric fluid. The in vitro study suggested that this system can float for more than 12 hrs and the in vivo study supported the in vitro floating characteristics of the hollow microspheres. Finding in the literature shows that the hollow microspheres are the promising drug delivery system to increase the gastric retention of the drugs.

Finally, with an increasing understanding of polymer behavior and role of biological factors, it is suggested that future research work in FDDS should be aimed at discovering means to accurately control the drug input rate into GIT for the optimization of the pharmacokinetic and toxicological profiles of the medicinal agents. Prolonged gastric retention and controlled delivery of the floating microspheres may offer numerous advantages, including improving bioavailability and therapeutic efficacy, and a possibility of reduction of dose size.

In vitro data obtained for floating microspheres of glipizide showed excellent floating ability, good buoyancy, and prolonged drug release. Microspheres of different size and drug content could be obtained by varying the formulation variables. Thus, the prepared floating microspheres may prove to be potential candidate for multiple unit delivery devices.

Therefore, it can be concluded that glipizide floating microspheres may be better candidate for once a day dosage form, after optimizing the various process parameters.

\section{ACKNOWLEDGMENT}

We are thankful to Micro-laboratories Pvt. Ltd., Kolkata, for providing generous gift of glipizide and Birbal Shahni Institute of Paleobotany, 
Lucknow, for providing Scanning Electron Facility (SEM). We also thank Head and Guide, Institute of Pharmacy, Bundelkhand University, Jhansi for providing infrastructure facility for the work.

\section{REFERENCES}

1. Seth PR, Tossounian J. The hydrodynamically balanced BBSTM: A novel drug delivery system for oral use. Drug Dev Ind Pharm 1984;10(2):313-39.

2. Moes AJ. Gasteopretentive dosage forms. Crit Rev Ther Drug Carrier Syst 1993;10(2):143-95.

3. Deshpande AA, Rhodes CT, Shah NH, Malick AW. Controlled release drug delivery system for prolonged gastric residence an overview. Drug Dev Ind Pharm 1996;22(6):531-9.

4. Sakagami M, Kinoshita W, Sakon K, Sato JI, Makino Y Mucoadhesive belomethasone microsphere for power inhalation: Their pharmacokinetics and pharmacodynamics evaluation. J Control Release 2002;80(1-3):207-18

5. Takishma J, Onishi H, Machida Y. Prolonged intestinal absorption of cephradine with chitosan-coated ethylcellulose microparticles in rats. Boil Pharm Bull 2002;25:1498-502.

6. Lim ST, Forbes B Berry DJ, Martin GP. Brown MB, In-vivo evaluation of novel hyaluronan/chitosan microparticulate delivery system for the nasal delivery of gentamicin in rabbits. Int J Pharm 2002;231(1):73-82.

7. Cuña M, Alonso MJ, Torres D. Preparation and in vivo evaluation of mucoadhesive microparticles containing amoxycillin-resin complexes for drug delivery to the gastric mucosa. Eur J Pharm Biopharm 2001;51:199-205

8. Deasy PB, Microencapsulation and Related Drug Process. New York: Marcel Dekker Inc.; 1984

9. Williams DA, Lemke TL, Williams L. Insulin and oral hypoglycemic drugs. In: Foye's Principles of Medicinal Chemistry. $5^{\text {th }}$ ed. New York: Lippincott Williams and Wilkins; 2002. p. 641-8.

10. Foster RH, Plosker GL. Glipizide. A review of the pharmacoeconomic implications of the extended-release formulation in Type 2 diabetes mellitus. Pharmacoeconomics 2000;18:289-306.
11. Kawashima Y, Niwa T, Takeuchi HT, Itoh Y. Preparation of multiunit hallow microspore with acrylic resin containing transilast and their drug release characteristic (in-vivo) and floating behavior (in-vivo). J Control Release 1991;16:279-90.

12. The United State Pharmacopeia XXIV. Rockville: United States Pharmacopoeial Convention; 2000. p. 1941-3.

13. Arshady R. Preparation of porous and nonporous biodegradable polymeric hallow microspheres. J Control Release 1991;17(1):1-22.

14. Kawashima Y, Niwa T, Takeuchi H, Hino T, Itoh Y. Hollow microspheres for use as a floating controlled drug delivery system in the stomach. J Pharm Sci 1992;81:135-40.

15. Zheng J, Liu C, Bao D, Zhao Y, Ma X. Preparation and evaluation of floating-bioadhesive microparticles containing clarithromycin or the eradication of Helicobacter pylori. J Appl Polymer Sci 2006;102:2226-32.

16. Price JC. Diffusion-controlled Systems: Polymeric Microcapsules, Edsln. Polymers for Controlled Drug Delivery. New York: Marcel Dekker, Inc.;1996. p. 256-67.

17. Paulo C, Jose MS. Modeling and comparison of dissolution profiles. Eur J Pharm Sci 2001;13(2):123-33

18. Desai SJ, Singh P, Simonelli AP, Higuchi WI. Investigation of factors influencing release of solid drug dispersed in wax matrices. III. Quantitative studies involving polyethylene plastic matrix. J Pharm Sci 1966;55(11):1230-4.

19. Higuchi T. Mechanism of sustained-action medication. Theoretical analysis of rate of release of solid drugs dispersed in solid matriceS. J Pharm Sci 1963;52:1145-9.

20. Gu X, Fediuk DJ, Simons FE, Simons KJ. Evaluation and comparison of five matrix excipients for the controlled release of acrivastine and pseudoephedrine. Drug Dev Ind Pharm 2004;30:1009-17.

21. Hixson AW, Crowell JH. Dependence of reaction velocity upon surface and agitation: Theoretical consideration. Ind Eng Chem 1931;23:923-31.

22. Kahn CR, Shechter Y. Oral hypoglycemic agents and the pharmacology of the endocrine pancreas. Goodman and Gilman's The Pharmacologica Basis of Therapeutics. Vol. 3. New York, NY: McGraw-Hill; 1991. p. 1461-95. 